(2) What is the neuroanatomical correlate of this clinical finding?

\title{
A developing visual field defect
}

(3) What further investigations should be performed?

Postgrad Med J 2002;78:106

\section{A J Larner}

\section{Answers on p 112.}

A 53 year old man complained of intermittent blurring of vision confined to the left eye over a period of 12 months. It did not stop him from reading or driving. There was no history of visual loss, eye pain, or double vision. He had recently been diagnosed with hypertension and hypercholesterolaemia, for which he was treated with felodipine ( $5 \mathrm{mg}$ once daily) and fluvastatin (40 mg once daily) respectively.

He attended his optician who performed a visual field test (suprathreshold static perimetry, fig 1), on the basis of which referral to a neurologist was recommended.

Clinical examination showed an uncorrected visual acuity of 6/9 left, 6/6 right. Reading pseudoisochromatic (Ishihara) plates he scored 2/17 left, 14/17 right. Confrontation testing of the visual fields showed no field loss to stationary or moving targets, but a red pin appeared less red in the left temporal field compared with the left nasal field; no such temporal desaturation to red was noted on the right. There was no relative afferent pupillary defect and fundoscopy was normal. There was no neglect of visual stimuli. The rest of the neurological examination was normal. Ophthalmological examination showed normal appearances of the ocular media, retina and optic nerve, and the intraocular pressures were normal.

\section{QUESTIONS}

(1) What is the visual field defect (fig l)?
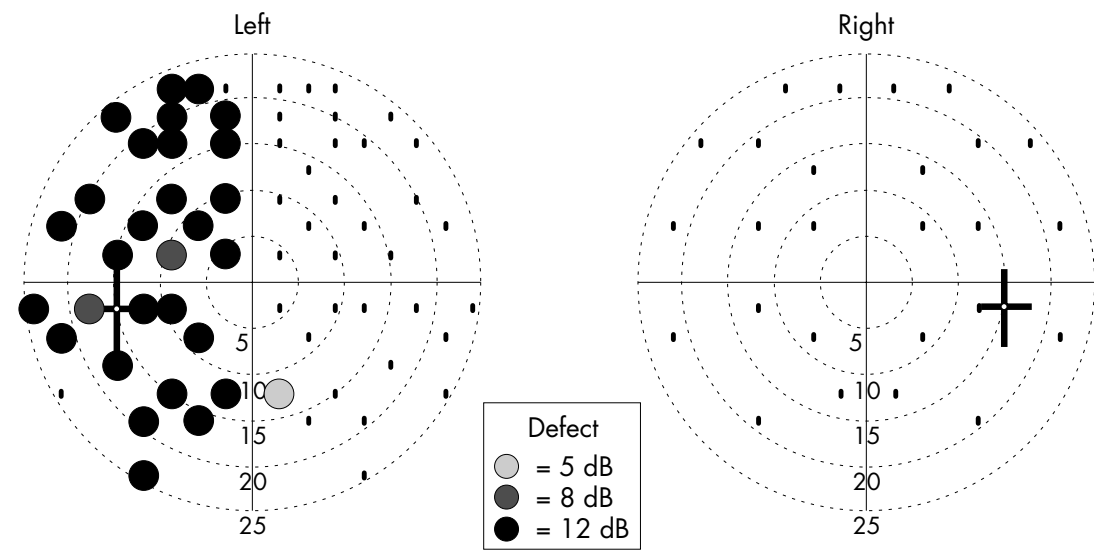

$$
\begin{gathered}
\text { Missed } / \text { presented }=30 / 65 \\
\text { False positives }=1 / 8 \\
\text { False negatives }=2 / 9 \\
\text { Threshold sensitivity }=23 \mathrm{~dB}
\end{gathered}
$$

\section{Author's affiliation}

A J Larner, Walton Centre for Neurology and Neurosurgery, Lower Lane, Fazakerley,

Liverpool L9 7L, UK

Correspondence to: Dr Larner; larner-a@wcnn-tr.nwest.nhs.uk

Submitted 20 November 2000

Accepted 15 February 2001

Figure 1 Visual fields mapped with suprathreshold static perimetry. 